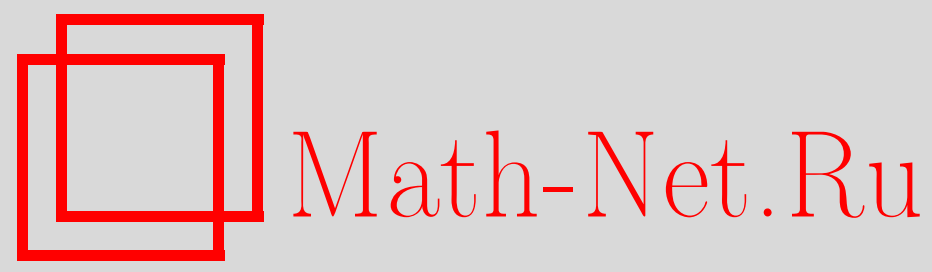

А. А. Майлыбаев, С. С. Григорян, О подготовительной теореме Вейерштрасса, Матем. заметки, 2001, том 69, выпуск 2, 194-199

DOI: https://doi.org/10.4213/mzm495

Использование Общероссийского математического портала Math-Net.Ru подразумевает, что вы прочитали и согласны с пользовательским соглашением http://www.mathnet.ru/rus/agreement

Параметры загрузки:

IP: 52.23 .180 .231

26 апреля 2023 г., 16:49:55

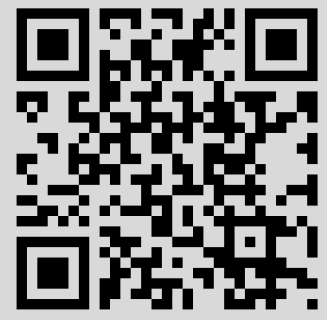




\title{
О ПОДГОТОВИТЕЛЬНОЙ ТЕОРЕМЕ ВЕЙЕРШТРАССА
}

\author{
С. С. Григорян, А. А. Майлыбаев
}

\begin{abstract}
Рассматривается подготовительная теорема Вейерштрасса, согласно которой аналитическая функция многих переменных, обращающаяся в некоторой точке в нуль, представима в окрестности этой точки в виде произведения ненулевой аналитической функции и полинома по одной из переменных. Коэффициенты полинома являются аналитическими функциями оставшихся переменных. В настоящей работе предлагается метод определения множителей (ненулевой функции и коэффициентов полинома) в виде рядов Тейлора, коэффициенты которых вычисляются при помощи явной рекуррентной процедуры по производньм исходной функции. В качестве приложения выведена явная формула, локально описывающая бифуркационную диаграмму, отвечающую двукратному корню, с точностью до членов второго порядка.

Библиография: 6 названий.
\end{abstract}

Рассмотрим аналитическую функцию многих переменных $f(z, \boldsymbol{p}), z \in \mathbb{C}, \boldsymbol{p}=\left(p_{1}, \ldots\right.$, $\left.p_{n}\right) \in \mathbb{C}^{n}$, которая обрашается в нуль в точке $z_{0}, \boldsymbol{p}_{0}=\left(p_{1}^{0}, \ldots, p_{n}^{0}\right)$. Согласно подготовительной теореме Вейерштрасса [1], [2] в окрестности точки $z_{0}, \boldsymbol{p}_{0}$ функция $f(z, \boldsymbol{p})$ представима единственным образом в виде произведения двух аналитических функций, одна из которых является полиномом по $z-z_{0}$ со старшим коэффициентом, равньм единице, а другая функция не равна нулю при $z=z_{0}, \boldsymbol{p}=\boldsymbol{p}_{0}$. Представление $f(z, \boldsymbol{p})$ в виде произведения двух множителей упрощает задачу исследования корней уравнения $f(z, \boldsymbol{p})=0$ и их свойств. Например, если $f(z, \boldsymbol{p})$ - полином по $z$, то из подготовительной теоремы Вейерштрасса следует, что можно существенно понизить порядок уравнения при исследовании зависимости от $\boldsymbol{p}$ отдельных корней полинома $f(z, \boldsymbol{p})$. Такая операция особенно полезна при анализе кратных корней в задачах устойчивости, где целью исследования является определение области устойчивости (множества значений параметров $\boldsymbol{p}$, при которых корни $z(\boldsymbol{p})$ удовлетворяют некоторому условию) [3]. Другой областью, где данная теорема может быть эффективно использована, является исследование бифуркационных диаграмм (множеств значений параметров $\boldsymbol{p}$, при которых имеются кратные корни).

Описанные вьше приложения осушествимы при условии, что множители, на которые по подготовительной теореме разлагается функция $f(z, \boldsymbol{p})$, известны. В настоящей работе предлагается метод определения этих множителей в виде рядов Тейлора, коэффициенты которых вычисляются явно по производным функции $f(z, \boldsymbol{p})$ в точке $z=z_{0}$,

Работа выполнена при поддержке Российского фонда фундаментальных исследований, грант РФФИ-ГФЕН № 99-01-39129, во время визита в Даляньский технический университет (Китай). 
$\boldsymbol{p}=\boldsymbol{p}_{0}$. В качестве приложения вьведена явная формула, локально описывающая бифуркационную диаграмму, отвечающую двукратному корню $z(\boldsymbol{p})$, с точностью до членов второго порядка.

Подготовительная теорема для случая бесконечно дифференцируемых функций была доказана Мальгранжем [4]. Результаты настоящей работы без изменений переносятся на этот случай и позволяют найти ряды Тейлора искомых фукнций.

ПОДГОТОВИТЕЛЬНАЯ ТЕОРЕМА ВЕЙЕРШТРАССА [1], [2]. Пусть аналитическая функиия $f(z, \boldsymbol{p})$ обращается в нуль при $z=z_{0}, \boldsymbol{p}=\boldsymbol{p}_{0}$, причем $z=z_{0}$ является т-кратным корнем уравнения $f\left(z, \boldsymbol{p}_{0}\right)=0$, m.e.

$$
f\left(z_{0}, \boldsymbol{p}_{0}\right)=\frac{\partial f}{\partial z}=\cdots=\frac{\partial^{m-1} f}{\partial z^{m-1}}=0, \quad \frac{\partial^{m} f}{\partial z^{m}} \neq 0
$$

где производные берутся при $z=z_{0}, \boldsymbol{p}=\boldsymbol{p}_{0}$. Тогда существует окрестность $U_{0} \subset \mathbb{C}^{n+1}$ точки $\left(z_{0}, \boldsymbol{p}_{0}\right)$, в которой функиия $f(z, \boldsymbol{p})$ может быть представлена в виде

$$
f(z, \boldsymbol{p})=\left(\left(z-z_{0}\right)^{m}+a_{m-1}(\boldsymbol{p})\left(z-z_{0}\right)^{m-1}+\cdots+a_{0}(\boldsymbol{p})\right) b(z, \boldsymbol{p}),
$$

где $a_{0}(\boldsymbol{p}), \ldots, a_{m-1}(\boldsymbol{p}), b(z, \boldsymbol{p})$ - аналитические функиии, однозначно определяемые функиией $f(z, \boldsymbol{p})$, причем $a_{i}\left(\boldsymbol{p}_{0}\right)=0, b\left(z_{0}, \boldsymbol{p}_{0}\right) \neq 0$.

Для осуществления разложения (1) необходимо определить функции $a_{i}(\boldsymbol{p})$ и $b(z, \boldsymbol{p})$. В окрестности точки $z=z_{0}, \boldsymbol{p}=\boldsymbol{p}_{0}$ функции $a_{i}(\boldsymbol{p})$ и $b(z, \boldsymbol{p})$ представимы в виде рядов Тейлора

$$
a_{i}(\boldsymbol{p})=\sum_{\boldsymbol{h}} \frac{1}{\boldsymbol{h} !} a_{i, \boldsymbol{h}} \Delta \boldsymbol{p}^{\boldsymbol{h}}, \quad b(z, \boldsymbol{p})=\sum_{k, \boldsymbol{h}} \frac{1}{k ! \boldsymbol{h} !} b_{k, \boldsymbol{h}} \Delta z^{k} \Delta \boldsymbol{p}^{\boldsymbol{h}}
$$

где суммы берутся по всем $k \in \mathbb{Z}_{+}, \boldsymbol{h}=\left(h_{1}, \ldots, h_{n}\right), h_{i} \in \mathbb{Z}_{+}\left(\mathbb{Z}_{+}-\right.$множество целых неотрицательных чисел), и использованы следующие обозначения:

$$
\begin{gathered}
a_{i, \boldsymbol{h}}=\frac{\partial^{|\boldsymbol{h}|} a_{i}}{\partial p_{1}^{h_{1}} \cdots \partial p_{n}^{h_{n}}}, \quad b_{k, \boldsymbol{h}}=\frac{\partial^{k+|\boldsymbol{h}|} b}{\partial z^{k} \partial p_{1}^{h_{1}} \cdots \partial p_{n}^{h_{n}}}, \quad|\boldsymbol{h}|=h_{1}+\cdots+h_{n}, \\
\Delta \boldsymbol{p}^{\boldsymbol{h}}=\prod_{j=1}^{n}\left(p_{j}-p_{j}^{0}\right)^{h_{j}}, \quad \Delta z^{k}=\left(z-z_{0}\right)^{k}, \quad \boldsymbol{h} !=h_{1} ! \cdots h_{n} !
\end{gathered}
$$

Все производные вычисляются при $z=z_{0}, \boldsymbol{p}=\boldsymbol{p}_{0}$. Под производной нулевого порядка подразумевается значение функции в точке, в частности, $b_{k, 0}=\partial^{k} b / \partial z^{k}$. Аналогично обозначаются производные функции $f(z, \boldsymbol{p})$. Определение функций $a_{i}(\boldsymbol{p}), b(z, \boldsymbol{p})$ эквивалентно нахождению производных $a_{i, \boldsymbol{h}}, b_{k, \boldsymbol{h}}$, определяюших коэффициенты разложения (2). В следующей теореме дается явное выражение для искомых производных через производные функции $f(z, \boldsymbol{p})$. 
ТЕОРемА. Производные $a_{i, \boldsymbol{h}}$ и $b_{k, \boldsymbol{h}}$ функиий $a_{i}(\boldsymbol{p}), b(z, \boldsymbol{p})$ в соотношении (1) удовлетворяют следующим рекуррентным соотношениям:

$$
\begin{aligned}
a_{i, \boldsymbol{h}} & =\sum_{j=0}^{i} \alpha_{i j} F_{j, \boldsymbol{h}}, \quad F_{j, \boldsymbol{h}}=f_{j, \boldsymbol{h}}-\sum_{k=0}^{j} \sum_{\substack{\boldsymbol{h}^{\prime}+\boldsymbol{h}^{\prime \prime}=\boldsymbol{h} \\
\boldsymbol{h}^{\prime} \neq 0, \boldsymbol{h}^{\prime \prime} \neq 0}} c\left(j, k ; \boldsymbol{h}^{\prime}, \boldsymbol{h}^{\prime \prime}\right) a_{k, \boldsymbol{h}^{\prime}} b_{j-k, \boldsymbol{h}^{\prime \prime}} \\
b_{k, \boldsymbol{h}} & =\frac{k !}{(m+k) !}\left[f_{m+k, \boldsymbol{h}}-\sum_{j=0}^{m-1} \sum_{\substack{\boldsymbol{h}^{\prime}+\boldsymbol{h}^{\prime \prime}=\boldsymbol{h} \\
\boldsymbol{h}^{\prime} \neq 0}} c\left(m+k, j ; \boldsymbol{h}^{\prime}, \boldsymbol{h}^{\prime \prime}\right) a_{j, \boldsymbol{h}^{\prime}} b_{m+k-j, \boldsymbol{h}^{\prime \prime}}\right]
\end{aligned}
$$

где коэффициенты $\alpha_{i j}(i \geqslant j), c\left(k, j ; \boldsymbol{h}^{\prime}, \boldsymbol{h}^{\prime \prime}\right)$ определяются соотношениями

$$
\begin{aligned}
\alpha_{j j}=\frac{m !}{j ! f_{m, 0}}, \quad \alpha_{i j} & =-\frac{m !}{f_{m, 0}} \sum_{k=j}^{i-1} \frac{f_{m+i-k, 0} \alpha_{k j}}{(m+i-k) !} \quad(i>j) \\
c\left(j, k ; \boldsymbol{h}^{\prime}, \boldsymbol{h}^{\prime \prime}\right) & =\frac{j !}{(j-k) !} \prod_{s=1}^{n} \frac{\left(h_{s}^{\prime}+h_{s}^{\prime \prime}\right) !}{h_{s}^{\prime} ! h_{s}^{\prime \prime !} !}
\end{aligned}
$$

ДОКАЗАТЕЛЬСТВо. Соотношения (4) для $\boldsymbol{h}=0$ имеют вид

$$
b_{k, 0}=\frac{k ! f_{m+k, 0}}{(m+k) !}
$$

Они получаются путем $(m+k)$-кратного дифференцирования по $z$ тождества $f\left(z, \boldsymbol{p}_{0}\right)=$ $\left(z-z_{0}\right)^{m} b\left(z, \boldsymbol{p}_{0}\right)$. Возьмем производную $\partial^{i+|\boldsymbol{h}|} / \partial z^{i} \partial p_{1}^{h_{1}} \cdots \partial p_{n}^{h_{n}}$ от обеих частей равенства (1):

$$
f_{i, \boldsymbol{h}}=\sum_{j=0}^{\min (i, m)} \sum_{\boldsymbol{h}^{\prime}+\boldsymbol{h}^{\prime \prime}=\boldsymbol{h}} c\left(i, j ; \boldsymbol{h}^{\prime}, \boldsymbol{h}^{\prime \prime}\right) a_{j, \boldsymbol{h}^{\prime}} b_{i-j, \boldsymbol{h}^{\prime \prime}}, \quad a_{m, \boldsymbol{h}^{\prime}}= \begin{cases}1, & \boldsymbol{h}^{\prime}=0 \\ 0, & \boldsymbol{h}^{\prime} \neq 0\end{cases}
$$

Пусть $i<m$. Выразив члены суммы, содержащие $a_{j, h}$, через остальные слагаемые и используя (6), получим

$$
\sum_{j=0}^{i} \beta_{i j} a_{j, \boldsymbol{h}}=F_{i, \boldsymbol{h}}, \quad \beta_{i j}=\frac{i ! f_{m+i-j, 0}}{(m+i-j) !}
$$

где величины $F_{i, \boldsymbol{h}}$ определены в $(3)$. Уравнения (8) для $i=0, \ldots, m-1$ можно записать в матричной форме

$$
\mathbf{G}\left(\begin{array}{c}
a_{0, \boldsymbol{h}} \\
\vdots \\
a_{m-1, \boldsymbol{h}}
\end{array}\right)=\left(\begin{array}{c}
F_{0, \boldsymbol{h}} \\
\vdots \\
F_{m-1, \boldsymbol{h}}
\end{array}\right), \quad \mathbf{G}=\left(\begin{array}{cccc}
\beta_{00} & 0 & 0 & 0 \\
\beta_{10} & \beta_{11} & 0 & 0 \\
\vdots & \vdots & \ddots & 0 \\
\beta_{(m-1) 0} & \beta_{(m-1) 1} & \cdots & \beta_{(m-1)(m-1)}
\end{array}\right) .
$$


Так как по условию $\beta_{i i}=i ! f_{m, 0} / m ! \neq 0$, то $\operatorname{det} \mathbf{G} \neq 0$ и, следовательно, система уравнений имеет единственное решение вида

$$
\left(\begin{array}{c}
a_{0, \boldsymbol{h}} \\
\vdots \\
a_{m-1, \boldsymbol{h}}
\end{array}\right)=\mathbf{G}^{-1}\left(\begin{array}{c}
F_{\boldsymbol{h}, 0} \\
\vdots \\
F_{\boldsymbol{h}, m-1}
\end{array}\right), \quad \mathbf{G}^{-1}=\left(\begin{array}{ccc}
\alpha_{00} & 0 & 0 \\
\vdots & \ddots & 0 \\
\alpha_{(m-1) 0} & \cdots & \alpha_{(m-1)(m-1)}
\end{array}\right) .
$$

То, что матрища $\mathbf{G}^{-1}$ имеет вид (9) с элементами $\alpha_{i j}$, определенными в $(5)$, можно доказать путем непосредственной проверки тождества $\mathbf{G G}^{-1}=\mathbf{I}(\mathbf{I}$ - единичная матрица). Равенство (9) представляет собой матричную запись соотношений (3) теоремы.

Рассмотрим случай $i \geqslant m$. Тогда соотношение (4) теоремы получается перестановкой членов в равенстве (7) с учетом (6) и при замене $i$ на $m+k$.

Соотношения (3)-(5) представляют собой рекуррентные соотношения, позволяющие находить производные функций $a_{i}(\boldsymbol{p}), b(z, \boldsymbol{p})$ по производным функции $f(z, \boldsymbol{p})$ в следующей последовательности: $b_{k, 0} \rightarrow a_{i, \boldsymbol{h}}, b_{k, \boldsymbol{h}}(|\boldsymbol{h}|=1) \rightarrow a_{i, \boldsymbol{h}}, b_{k, \boldsymbol{h}}(|\boldsymbol{h}|=2) \rightarrow \cdots$. При этом для определения производной $a_{i, \boldsymbol{h}}$ необходимо вычислить на предыдущих этапах значения $a_{j, \boldsymbol{h}^{\prime}}, b_{j, \boldsymbol{h}^{\prime}}$ для $j \leqslant i+\left(\left|\boldsymbol{h}-\boldsymbol{h}^{\prime}\right|-1\right) m$ и $\boldsymbol{h}^{\prime}<\boldsymbol{h}\left(h_{s}^{\prime} \leqslant h_{s}\right.$ для всех $s=1, \ldots, n$ и $h_{s}^{\prime}<h_{s}$ для некоторого $\left.s\right)$. Таким образом, значение $a_{i, \boldsymbol{h}}$ определяется производными $f_{k, \boldsymbol{h}^{\prime \prime}}$, где $k \leqslant i+\left|\boldsymbol{h}-\boldsymbol{h}^{\prime \prime}\right| m$ и $\boldsymbol{h}^{\prime \prime} \leqslant \boldsymbol{h}$.

Первые шаги рекуррентной процедуры имеют вид

$$
\begin{gathered}
a_{i}\left(\boldsymbol{p}_{0}\right)=0, \quad \frac{\partial^{k} b}{\partial z^{k}}=\frac{k !}{(m+k) !} \frac{\partial^{m+k} f}{\partial z^{m+k}}, \quad \frac{\partial a_{i}}{\partial p_{s}}=\sum_{j=0}^{i} \alpha_{i j} \frac{\partial^{j+1} f}{\partial z^{j} \partial p_{s}} \\
\frac{\partial^{k+1} b}{\partial z^{k} \partial p_{s}}=\frac{k !}{(m+k) !}\left[\frac{\partial^{m+k+1} f}{\partial z^{m+k} \partial p_{s}}-\sum_{j=0}^{m-1} \frac{(m+k) !}{(m+k-j) !} \frac{\partial a_{j}}{\partial p_{s}} \frac{\partial^{m+k-j} b}{\partial z^{m+k-j}}\right], \\
\frac{\partial^{2} a_{i}}{\partial p_{s} \partial p_{t}}=\sum_{j=0}^{i} \alpha_{i j}\left[\frac{\partial^{j+2} f}{\partial z^{j} \partial p_{s} \partial p_{t}}-\sum_{k=0}^{j} \frac{j !}{(j-k) !}\left(\frac{\partial a_{k}}{\partial p_{s}} \frac{\partial^{j-k+1} b}{\partial z^{j-k} \partial p_{t}}+\frac{\partial a_{k}}{\partial p_{t}} \frac{\partial^{j-k+1} b}{\partial z^{j-k} \partial p_{s}}\right)\right] .
\end{gathered}
$$

ПРИмЕР. Функцию $f(z, \boldsymbol{p})$ можно рассматривать как семейство функций одной переменной $z$, где $\boldsymbol{p}$ является вектором параметров. Бифуркационной диаграммой называется множество значений вектора $\boldsymbol{p}$, при которых функция $f(z, \boldsymbol{p})$ имеет кратные корни $z$. Рассмотрим двукратньй корень $z=z_{0}$. Из подготовительной теоремы Вейерштрасса следует, что поверхность в пространстве $\boldsymbol{p}$, на которой этот корень остается двукратньп (при этом значение корня может меняться), определяется равенством нулю дискриминанта $D=\left(a_{1}(\boldsymbol{p})\right)^{2}-4 a_{0}(\boldsymbol{p})$ уравнения $\left(z-z_{0}\right)^{2}+a_{1}(\boldsymbol{p})\left(z-z_{0}\right)+a_{0}(\boldsymbol{p})=0$. Подставив разложения (2) в уравнение $D=0$, запишем уравнение этой поверхности в виде

$$
\begin{aligned}
D= & -4 \sum_{r=1}^{n} \frac{\partial a_{0}}{\partial p_{r}}\left(p_{r}-p_{r}^{0}\right) \\
& +\sum_{s, t=1}^{n}\left(\frac{\partial a_{1}}{\partial p_{s}} \frac{\partial a_{1}}{\partial p_{t}}-2 \frac{\partial^{2} a_{0}}{\partial p_{s} \partial p_{t}}\right)\left(p_{s}-p_{s}^{0}\right)\left(p_{t}-p_{t}^{0}\right)+o\left(\left\|\boldsymbol{p}-\boldsymbol{p}_{0}\right\|^{2}\right)=0
\end{aligned}
$$


где $\|\boldsymbol{p}\|$ - норма в $\mathbb{C}^{n}$; первые и вторые производные функций $a_{0}(\boldsymbol{p}), a_{1}(\boldsymbol{p})$ выражаются через производные функции $f(z, \boldsymbol{p})$ по формулам (10); коэффициенты $\alpha_{i j}$ согласно $(5)$ определяются соотношениями

$$
\alpha_{00}=\alpha_{11}=\frac{2}{\partial^{2} f / \partial z^{2}}, \quad \alpha_{10}=-\frac{2}{3} \frac{\partial^{3} f / \partial z^{3}}{\left(\partial^{2} f / \partial z^{2}\right)^{2}} .
$$

Уравнениями (10)-(12) поверхность $D=0$ описывается в явном виде через производные функции $f(z, \boldsymbol{p})$ с точностью до членов второго порядка. Аналогичньм способом могут быть получены соотношения, описывающие бифуркационную диаграмму в окрестности корня произвольной кратности.

Бифуркационная диаграмма семейства функций $f(z, \boldsymbol{p})$ имеет физический смысл. Пусть $f(z, \boldsymbol{p})$ - многочлен по $z$, возникающий после замены $z=-\lambda^{2}$ из характеристического полинома линейной гамильтоновой или неконсервативной (циркуляционной) механической системы. Тогда часть бифуркационной диаграммы, характеризуемая положительными вещественными корнями, является гранищей области устойчивости, отвечающей колебательной форме потери устойчивости (флаттеру) [5], [6]. При этом соотношение (11) описывает границу области устойчивости локально с точностью до членов второго порядка.

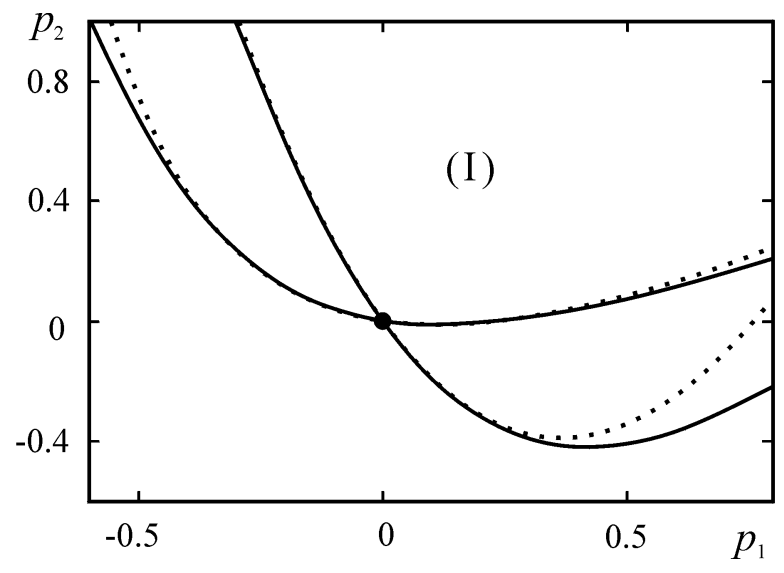

Рис. 1. Бифуркационная диаграмма семейства полиномов и ее аппроксимация

Для примера рассмотрим двухпараметрическую полиномиальную функцию

$$
f(z, \boldsymbol{p})=z^{5}-13 z^{4}+\left(62+\frac{1}{4} p_{1}\right) z^{3}-\left(134+p_{1}^{2}\right) z^{2}+\left(129+p_{2}\right) z-45+p_{1} p_{2} .
$$

При $\boldsymbol{p}_{0}=(0,0)$ полином $f\left(z, \boldsymbol{p}_{0}\right)$ имеет двукратные корни $z=1, z=3$ и простой корень $z=5$. Следовательно, бифуркационная диаграмма в окрестности точки $\boldsymbol{p}_{0}=(0,0)$ состоит из двух кривых. Эти кривые задаются уравнением $D=0$, записанньп в точках $\left(z_{0}, p_{1}^{0}, p_{2}^{0}\right)=(1,0,0)$ и $(3,0,0)$. Используя соотношения $(10)-(12)$, найдем уравнения кривьх $D=0$ с точностью до членов второго порядка

$$
\begin{aligned}
& z_{0}=1: \frac{1}{16} p_{1}+\frac{1}{4} p_{2}-\frac{15595}{65536} p_{1}^{2}+\frac{2525}{8192} p_{1} p_{2}+\frac{229}{4096} p_{2}^{2}=0 \\
& z_{0}=3: \frac{27}{8} p_{1}+\frac{3}{2} p_{2}-\frac{13815}{4096} p_{1}^{2}+\frac{661}{512} p_{1} p_{2}+\frac{49}{256} p_{2}^{2}=0
\end{aligned}
$$


Точный вид бифуркационной диаграммы и ее аппроксимация (13) изображены на рис. 1 сплошной и точечной линиями соответственно. Область (I) на рис. 1 , определяемая неравенствами $D>0$, отвечает наличию лишь вещественных корней $z(\boldsymbol{p})$. Отметим, что приближенные уравнения для бифуркационной диаграммы (13) были вычислены по значениям производных функции $f(z, \boldsymbol{p})$ в точках $\left(z_{0}, p_{1}^{0}, p_{2}^{0}\right)=(1,0,0)$ и $(3,0,0)$.

\section{СПИСОК ЦИТИРОВАННОЙ ЛИТЕРАТУРЫ}

[1] Weierstrass K. Einige auf die Theorie der analytischen Functionen mehrerer Veränderlichen sich beziehende Sätze // Mathematische Werke, II. Berlin: Mayer und Müller, 1895. P. 135-188.

[2] Шабат Б. В. Введение в комплексный анализ, Ч. ІІ. М.: Наука, 1976.

[3] Майлыбаев А. А. Об устойчивости полиномов, зависящих от параметров // Изв. РАН. Сер. Теория и системы управления. 2000. № 2. С. 5-12.

[4] Мальгранж Б. Подготовительная теорема для дифференцируемых функций // Особенности дифференцируемых отображений. Сборник статей. М.: Мир, 1968. С. 183-189.

[5] Арнольд В.И., Козлов В.В., Нейштадт А.И. Математические аспекты классической и небесной механики // Итоги науки и техники. Современные проблемы математики. Т. 3. М.: ВИНИТИ, 1985.

[6] Сейранян А.П. Бифуркации в однопараметрических циркуляционных системах // Изв. РАН. Сер. Механика твердого тела. 1994. № 1. С. 142-148.

Институт механики Московского государственного университета 